\title{
Immunology of biodegradable nanoparticles: a brief overview on a wide growing field
}

\author{
Anissa Pisani ${ }^{1,2}$, Giuseppe Bardi ${ }^{1}$ (1) \\ ${ }^{1}$ Nanobiointeractions and Nanodiagnostics, Istituto Italiano di Tecnologia, 16163 Genova, Italy \\ ${ }^{2}$ Department of Chemistry and Industrial Chemistry, University of Genova, 16146 Genova, Italy
}

*Correspondence: Giuseppe Bardi, Nanobiointeractions and Nanodiagnostics, Istituto Italiano di Tecnologia, Via Morego 30 , 16163 Genova, Italy. giuseppe.bardi@iit.it

Academic Editor: Wangxue Chen, National Research Council Canada, Canada

Received: February 19, 2021 Accepted: April 11, 2021 Published: April 30, 2021

Cite this article: Pisani A, Bardi G. Immunology of biodegradable nanoparticles: a brief overview on a wide growing field. Explor Immunol. 2021;1:48-60. https://doi.org/10.37349/ei.2021.00006

\begin{abstract}
Immunity is continuously evolving by evolutionary mechanisms shaped by pathogenic stimuli of different kinds. Man-made nanomaterials (NMs) have been developed in the last decades and represent a novel challenge for our immune system, especially when applied to medical science. Toxicological studies of such nanoparticles (NPs) revealed that size, shape, and surface chemistry are key parameters to understand their noxious effects on cellular mechanisms. Less is known on the immune reactions to NMs since prolonged exposure data are not so detailed as the results for acute administration. The importance of immunity to biocompatible NPs is underlined by their increasing use as drug or gene delivery carriers in common pharmaceutical preparations and vaccines. In the latter case, the immunomodulatory properties of NMs allow their use also as efficient adjuvants to enhance the innate immune response. In the current manuscript, the authors discuss the main concepts in this fast-growing field by restricting our view to NMs with consolidated application in biomedicine.
\end{abstract}

\section{Keywords}

Immune system, nanoparticles, inflammation, cytokines, protein corona

\section{Introduction}

In the past decades the development of diverse nanotechnologies allowed their application in several fields including medicine, mainly for drug delivery and diagnostics [1]. The physicochemical diversity of nanomaterials (NMs) extends far beyond the conditions of biocompatibility that allow a profitable use of NMs in medicine. For instance, most of the inorganic or carbon-based materials are not degradable and poorly soluble rising concerns for tissue accumulation and cytotoxicity [2]. High doses of inorganic nanoparticles (NPs) disrupt the physiological mechanisms inducing toxicity, whereas lower doses could not show direct noxious effects hiding long-term events appearing at a later stage. NP interaction with the 
immune system (IS) deserves special attention, as NPs could evoke potential inflammatory responses that need appropriate investigation before any biomedical claim [3].

NP size, shape and chemical surface determine their recognition by cells and molecules of the IS. In particular, the different surface chemistry plays a major role in the interactions with the biological environment and often defines NP immune-compatibility [4]. Continuous research is currently focused on NM coatings with the ability to hide from the IS and delay their clearance once released, for example, in the bloodstream [5]. One of the most common strategy is the modification of NM surface with polymers such as poly(ethylene glycol) (PEG) or poly(2-oxazoline) (POx) to improve drug delivery systems and diagnostic nano-tools. Though several chemical technologies have been developed to avoid IS, many researchers are currently trying to stimulate an immune reaction by tailored inorganic or organic NPs for specific adjuvant activity in vaccine delivery [6]. A variety of NMs show amazing drug/gene delivery performances or immunomodulatory properties [7]. Some of them already have a key role in current therapies, especially in severe acute respiratory syndrome coronavirus 2 (SARS-CoV-2) vaccine delivery [8, 9], whereas others show potential applications aimed at interfering with abnormal immune reactions like coronavirus disease 2019 (COVID-19)-induced cytokine storm [10].

All these evidences emphasize the need for a deep investigation of NM-mediated immune responses to unravel their immune-toxicity mechanisms and design safe particles for medical applications. In the present review we restrict our attention to some prototype biodegradable NPs and their potential or documented inflammatory reactions.

\section{Immune reactions to polymer NPs}

Several and diverse polymers can be assembled in nanostructures. They are divided in natural [11] or synthetic polymers [12] with a huge range of characteristics and possible modifications. Natural polysaccharides, most of which extracted from invertebrates, algae, fungi or bacteria, are frequently used to prepare stable NPs for applications in biomedical research [13]. As repetitive and conserved patterns, polysaccharides could be recognized by the innate IS cells through pathogen-associated molecular patterns (PAMPs) recognition receptors. Specific responses against exogenous sugars or glycosaminoglycans are quickly mounted by first reacting phagocytes, namely neutrophils and macrophages. These cells show a wide set of pattern recognition receptors (PRR) types, such as Toll-like receptors (TLRs), C-type lectin receptors (CLRs), retinoic acidinducible gene (RIG)-I-like receptors (RLRs), and nucleotide-binding oligomerization domain (NOD)-like receptors (NLRs). Most of their signal pathways converge on the nuclear factor (NF)- $\kappa B$ transcription factor inducing the expression of genes coding for pro-inflammatory mediators, including cytokines, chemokines, antimicrobial proteins and type I interferons (IFN). A first raised question with controversial answers is whether natural polysaccharides folded to form nanostructures are still able to interact with immune receptors [13]. Within the vast families of natural polymers, we will consider only a couple of them as model for commonly induced immune effects.

Monocyte response to starch NPs, for example, strictly depends on the fine differences of their specific saccharide composition [14]. In one of our studies, we showed that NPs of similar size (from 40 $\mathrm{nm}$ to $100 \mathrm{~nm}$ ) prepared with starches extracted from two different potatoes, (i.e. Solanum tuberosum and Solanum goniocalyx), induced a different response. THP-1 cells released the pro-inflammatory cytokines interleukin (IL)-6, IL-1 $\beta$, tumor necrosis factor (TNF)- $\alpha$ and chemokines (C-C motif) ligand 2 (CCL2), CCL4, and CCL5 in the presence of NPs synthesized with starch extracted from S. goniocalyx. On the contrary, this monocytic cell line did not show any significant pro-inflammatory reaction towards $S$. Tuberosum starch-NPs. Since the starch is formed by amylose and amylopectin molecules, structural differences may be due to a dissimilar molecular branching and folding in the particle. Supported by previous results on starch films [15], we hypothesized that fine polysaccharide changes may induce the recruitment of different PRRs on THP-1 membranes and a different cytokine response. Starch-NPs' properties depend on the botanical source of the polymer and drive NP-employment in nano-formulations aimed at stimulation (e.g., adjuvants) or avoidance of inflammatory reactions (e.g., drug delivery) [16]. In the first case, it would be always important to understand 
the extent of the inflammatory response with in vivo studies. Unfortunately, no such studies have been already done using starch NPs. However, results from in vitro experiments indicate that pro-inflammatory IL-6, TNF- $\alpha$ and IL-1 $\beta$ are also released by mixed monocytes and lymphocytes cultures when blends of corn starches are administered for long time [17].

Chitin is another very abundant natural polysaccharide used for NP preparation. It is a common component of insects and crustaceans' exoskeleton, also present in some fungi. Even if it is proved that sixsubunit-long oligomers of fungal chitin bind to TLR2 [18], it is not clear whether the same molecule folded and arranged in a particle structure still maintains its potential inflammatory and allergy-promoting PAMP [19]. As purified, chitin is hydrophobic and insoluble, so its deacetylated derivative chitosan, which is soluble in weak acidic solutions, is preferred to prepare NPs [20]. Many aspects of chitin/chitosan NP effects on the IS still need to be elucidated, nevertheless, data regarding enhanced cytokine release by different immune cells have been shown in splenocytes of immunized mice with ovalbumin [21]. Especially, mRNA expression of IL-2, IFN-g and IL-10 promoting both T-helper (Th)1 and Th2 adaptive immune responses. Based on these evidences, chitosan and other polysaccharide NPs are proposed as natural nano-adjuvants in different studies [22]. Chitosan has a wide range of application in pharmaceutical sciences and tissue engineering $[23,24]$. Its charge varies in media with different $\mathrm{pH}$ as its amino groups are protonated. Chitosan in such condition becomes a cationic polymer strongly interacting with several molecules in the biological milieu. Actually, NP interactions in biological media mainly depends on their chemical surface and this aspect will be carefully described in the following text.

Unlike natural polymers, synthetic ones can be designed with exclusive intrinsic properties for specific medical applications [25]. NPs prepared with these polymers are biodegradable, increase the stability and bioavailability of several drugs, often providing their controlled release. Food and Drug Administration (FDA) already approved different synthetic polymers, such as poly( $\varepsilon$-caprolactone) (PCL), poly(lactide acid) (PLA), and poly(lactic-co-glycolic acid) (PLGA), [26-28]. In general, NPs synthesized with FDA-approved polymers are harmless, albeit some indications reveal that particle size can be crucial to provoke inflammatory signaling in vitro [29]. Nicolete and colleagues [29] showed that PLGA particles of micrometric diameter strongly interact with cell membrane protein receptors inducing NF- $\kappa \mathrm{B}$ dependent inflammatory stimulus after their uptake in murine macrophages. Conversely, nano-sized particles of the same bulk material do not show immune-toxicity. This effect may be due to the interactions with more proteins onto the cell surface and the different internalization pathway engaged.

Crucial indirect NP-mediated immunomodulatory effects depend on the adsorption of different molecules onto their surfaces. For instance, PLA-NP chemical affinity for Vitamin E or TLR agonists have been recently studied [30]. Polymers' ability to adsorb specific molecules is very important to modulate the immune responses. The set of molecules adsorbed onto the NP surface, mainly proteins in the blood or other biological fluids, is called protein corona (PC) [31]. PC can be divided in "hard" and "soft" corona. The first is formed by proteins tightly bound to the chemical surface, whereas the soft-corona is characterized by polypeptides interacting through reversible binding in the outer layer of the PC. NP surface charge plays an essential role in PC configuration by selecting the protein content [32,33]. Specific passive NP surface functionalization can be achieved by tailoring the polymer chemistry [34]. In turn, the presence of the molecular coating may affect the expected NP release of drug or vaccines [35]. The possibility to reduce the therapeutic efficiency make PC-induced immunomodulation a fundamental challenge for pre-clinical and clinical research [36].

Particularly, NPs released in the bloodstream face their opsonization by complement proteins and immunoglobulins [37]. Experiments with dextran coated NPs revealed that C3 can covalently bind the coronaproteins inserted into the dextran shell starting the complement cascade [38]. Alternatively, immunoglobulins can directly bind PC proteins with the creation of enzymatically active complexes antibody-C3b [39]. The final result of NP opsonization is the recognition by complement or immunoglobulin receptors on the phagocytes followed by their activation.

PEGs is widely used to reduce molecule adsorption and increase the circulation time of bioactive compounds [40]. PEG is present in several forms in linear or branched chains of ethylene glycol-monomers 
with different molecular weights (from 2,000 Da to $20 \mathrm{kDa}$ ) and shapes. For long time, PEG has been considered a key coating polymer to hide drugs or NPs from the IS. Unfortunately, B cell production of anti-PEG antibodies is emerging as major concern for the reduced efficacy of PEGylated therapeutics and the potential adverse effects of immune reactions [41-44]. Blood samples from patients treated with PEG conjugated drugs revealed the presence of anti-PEG immunoglobulin (Ig)M and IgG. Surprisingly, anti-PEG IgM have also been found in healthy untreated individuals [42]. Antibody binding leads to accelerated blood clearance (ABC phenomenon) of the PEGylated drug. This effect is recurrent when further PEG doses are administrated. In addition, anti-PEG IgE production or specific IgG can induce immediate hypersensitivity or complement-activation-related pseudo-allergy (CARPA) posing further concerns for prolonged clinical use $[45,46]$. Reasonably, inflammatory responses involving innate and adaptive reactions can be thought for PEGylated NPs with different core materials. Full-polymer NPs, for instance PEG-PLGA NPs, are not recognized by Th lymphocytes eluding a T cell-dependent B cell antibody production and enhancement. On the other hand, the prediction of enhanced Th cell-dependent humoral responses is not unrealistic for PEGprotein NPs, as recently observed after administrations of PEG conjugated protein drugs [41]. Theoretically, following internalization of PEGylated protein-NPs, macrophages, or dendritic cells (DCs) could present NP-core peptides onto the major histocompatibility complex (MHC) II protein complex and activate Th lymphocytes. As well, B lymphocytes recognizing PEG on the NP surface through membrane B cell receptor (BCR) could enhance Th cells pre-stimulated with the processed core peptides. This hypothesis is reinforced by several clinical reports of a robust production of treatment-induced anti-PEG Abs against PEGylated drugs compromising their therapeutic efficacy and increasing the adverse effects [43]. It is worth to mention that alternatives to PEG are under intensive research, as an increasing amount of anti-PEG antibodies is currently seen in the population maybe related to the growing use of PEG in therapeutics and beauty products (e.g., body creams) [47].

\section{Protein NPs recognition by the IS}

Full-protein NPs are, anyhow, considered advantageous for drug delivery applications due to their biodegradability, natural abundance and cost-effective synthesis process [48]. Indeed, several challenges encountered with different materials in the biological milieu, like solubility and PC, can be overcome by using NP proteins from the same environment. Nevertheless, assembling peptides in nano-scale structures may induce irregularly folded protein-domains recognized as non-self and activating complement opsonizationmediated sequestration by phagocytes.

Enhanced immune responses towards peptide-NPs can be used advantageously to prepare biodegradable adjuvant NPs for vaccine technology. For example, virus like particles (VLPs) are nanometric particles of viral-capsid proteins able to prime our defense mechanisms almost like the original hostile viruses [49, 50]. VLP derived peptides are efficiently presented by both MHC I and MHC II molecules allowing CD8 ${ }^{+}$and $\mathrm{CD} 4^{+}$lymphocytes responses. As well, the activation of PRRs on the macrophage surface induce the release of inflammatory cytokines enhancing the reaction. Even in the absence of MHC II-dependent CD4 ${ }^{+} \mathrm{T}$ cell activation, the highly repetitive arrangements of VLP surface proteins can facilitate BCR crosslinking. This multiple epitope engagement overcomes the triggering threshold in the absence of Th lymphocyte support and may induce a long-lasting humoral response.

NPs with different core materials could also be decorated with viral proteins [51]. Depending on the chosen proteins, the functionalized surface can determine NP fate, its cell targeting or mediate acquired stealth properties [52]. Surface modification with self-ligands of immune receptors can also be a method to increase cell targeting and particle circulation [53]. We recently focused on chemokine-functionalized NPs to improve cell targeting [54] and impair CXCR4-mediated cell migration [55]. Our interest on chemokine NP decoration is motivated by the versatility of cell targets in different inflammatory conditions. As chemokine receptor expression-pattern depends on the physiological state, we aim at designing NPs able to bind immune cells driven by immune mediators. Roughly 50 human chemokines binding 10 different receptors 
are known today [56]. This scenario offers various opportunities to interact with most of the immune cells in their different state of activation.

\section{Lipid NPs and current therapeutic opportunities}

Liposomes have been developed more than fifty years ago [57], but still prove to be versatile and functional NP-drug carriers [58]. They are spherical vesicles made of a single or multiple lipid-layer surrounding an aqueous core, commonly prepared in different sizes (0.05-5.0 $\mu \mathrm{m}$ in diameter). They are composed of natural or synthetic lipids, usually phospo- and sphingo-lipid often containing other constituents such as cholesterol or hydrophilic polymer-conjugated lipids. The concomitant presence of hydrophobic bilayer (with inner and outer polar heads) and hydrophilic core allows the encapsulation of lipophilic or hydrophilic drugs in the phospholipid bilayer or within the aqueous core, respectively. Like other NPs, liposomes have different interactions with the IS on the basis of their physicochemical properties, [59]. Since early studies in vivo, macrophage phagocytosis of liposome has been observed as the major event after their release in the bloodstream [60,61]. In particular, composition [62], size and surface charge [63] were described as crucial feature determining liposome interaction with serum proteins and the following phagocyte uptake. Macrophages in different tissues tend to uptake liposomes with different surface charge with a different efficiency [64]. This event was shown by Aramaki and colleagues [64] more than 25 years ago demonstrating that PC and surrounding molecules determine the fate of liposomes with dissimilar composition/charge surface. They demonstrated that peritoneal macrophages significantly internalized negatively charged liposomes, containing high concentration of phosphatidylserine. On the contrary, splenic macrophages took up positively charged liposomes with increased internalization depending on their stearylamine concentration. The opsonization of stearylamine-containing liposomes by fibronectin was crucial to enhance their uptake by splenic macrophages. Intriguingly, it did not improve the internalization by peritoneal phagocytes independently of their surface charge. The role of liposome composition-dependent opsonization was deepened by studies on complement proteins, immunoglobulins, fibronectin and apo-lipoproteins [63, 65, 66], comprehensively described by Zahednezhad et al. [59].

Different observations revealed that negatively charged liposomes activate the classical pathway binding to IgG, IgM and C1q or C-creative protein (CRP). Conversely, positively charged liposomes can activate the alternative pathway after opsonization by C3b, IgG or C4b2a3b. The presence of cholesterol in the liposome composition can also influence complement activation, namely, high cholesterol content is associated with maximal activation [67-70].

PEGylation is a common strategy to hide liposomes from the IS, although this chemical modification can rise the concerns previously mentioned for the therapeutic use of PEGylated NPs (i.e. PEG immunogenicity, ABC phenomenon, C-protein activation and CARPA effect) [71].

The innate IS activation triggered by liposomes could also feasibly lead to an acquired immune response. The activation of innate immune cells through PRRs, TLRs and the mannose receptor (CD206), such as DCs and natural killer cells (NKCs), gives rise to adaptive response with several applications in cancer or infectious disease research. Liposomes modification targeting PRRs has been experimentally proved as an efficient strategy for both cancer immune-therapy and in immune-vaccine development [72, 73]. Cationic liposomes loaded with tumor-specific synthetic peptides and an adjuvant TLR3-ligand intradermally injected in mice with melanoma and human papillomavirus (HPV)-induced tumors significantly activated antigenspecific $\mathrm{CD}^{+}$and $\mathrm{CD} 4^{+}$cells causing target cell cytotoxicity and controlling tumor outgrowth. Other examples of liposome versatility as adjuvant drug carrier have been described for folate-coated liposomes containing zoledronic acid, selectively taken up through folate receptor-mediated endocytosis in tumor associated macrophages [74]. Diverse immune pathways can be specifically activated by tailoring liposome composition, functionalization, and active molecule cargoes. For instance, a liposome-vaccine incorporating diphtheria toxoid and the TLR3-ligand poly inosinic:polycytidylic acid (poly I:C) in an ovine lymphatic cannulation model provided activation of the innate IS, TLR signaling and inflammasome pathway [75]. Up-regulation of IL-17A and IL-17F also suggested the role of Th17 lymphocytes in the earliest adaptive response to the 
vaccine. In another study a liposome formulation containing TLR4 and TLR7/8 agonists was able to generate intestinal IgA, plasma IgG2a/IgG1, IFN $\gamma$ and IL-17A promoting a powerful mucosal immune response against the Entamoeba histolytica Gal/GalNac lectin LecA antigen [76]. Further examples of liposome NPs preclinical applications are shown by mannosylated liposome-protamine-DNA carriers [77] which are able to induce significant up-regulation of CD80, CD86 and CD40 in primary bone marrow-derived DCs. This is an advantageous boosting of T lymphocytes mediated response through CD28 and CD40-ligand improving their carrier activity for DNA vaccines.

Much emphasis on liposome formulations aimed at vaccination purposes derives from the anti-SarsCov2 vaccine launched by Pfizer. The vaccine contains a PEGylated liposome entrapping the mRNA encoding for the SarsCov2 capsid-protein Spike2. This formulation is characterized by fast release and translation of the cargo mRNA. The translated spike 2 protein is quickly recognized by the patrolling immune cells and further processed. The vaccine is able to elicit both humoral and Th1 mediated response reaching almost 95\% protection to COVID-19, although immunization lasting time still need further data collection [78-80]. Based on the first indications coming from the current vaccination campaign around the world, no side effects associated with the liposome carrier have been reported. A very recent publication, however, report cases of lymphadenopathy after vaccination in Israel [81]. Since no further data are available yet, and the affected lymph nodes are the ones closed to the injection, we hypothesis that a fast liposome degradation might bust the cargo-mRNA transcription and the following immune response. However, the enlarged nodes recovered the normal size in a few days. Rare allergic reactions to vaccine-liposome PEGylated lipids have been also reported [82]. The authors suggest an IgE-mediated reaction, without excluding the hypothesis of CARPA, a common effect previously described as consequence of other types of NP PEGylation.

As previously mentioned, an improving strategy could be the use of similar but less antigenic polymers to coat the liposomal surface. Among them, a just published study regarding solid tumor treatments using liposome drug carriers compared PEGylated thermosensitive liposome versus 1,2-dipalmitoyl-sn-glycero3-phosphodiglycerol (DPPG2) modified ones [83]. DPPG2-liposomes demonstrated decreased complement activation and granulocyte interaction making them interesting nano-tools to be further investigated.

Lipid NPs (LNPs) are NPs similar to liposome, consisting of a lipid matrix stabilized by surfactants and not displaying a lipid bilayer surrounding the aqueous core [84]. They are made with physiologic lipids, such as purified triglycerides, fat acids and steroids, with a size range of 10-500 nm. In particular solid LNPs (SLNPs) are largely investigated as gene delivery vehicles taking advantage of cationic lipids that can complex with the negatively charged nucleic acids $[85,86]$. As well as for liposomes, major interest is reserved to SLNPs to improve the stability of mRNA and siRNA vaccine formulation [87]. Lutz and colleagues [87] report that a single intramuscular dose of LNP-formulated mRNA encoding rabies or influenza antigens induce protective and stable antibody titers in non-human primates, lasting up to one year. SLNPs are also applied to therapeutic delivery in cancer. siRNA targeting the vascular endothelial growth factor (VEGF), which is involved in tumor angiogenesis, and the metastasis mediator kinesine spindle protein (KSP) promoted target downregulation, antitumor activity and regression of liver metastasis in endometrial cancer [88].

LNP efficiency can be improved by PEGylation or specific composition, such as the use of ionizable aminolipids to form small multilamellar vesicle structures [89]. As for the other described biodegradable NPs, immunotoxicity, pro-inflammatory cytokines production, CARPA and eventually, anaphylactic shock are mainly ruled by the surface chemistry determining their PC and tissue-specific molecule adhesion [90, 91].

Summarized in Table 1 are reported cell receptor engaged, signal pathways and cytokine released by biodegnadable NPs used in biomedicine. 
Table 1. Biodegradable NP-induced pathways

\begin{tabular}{|c|c|c|c|c|}
\hline Biodegradable NPs* & Target receptors & Reported signal pathways $\S$ & Reported protein expression $\S$ & References \\
\hline \multicolumn{5}{|l|}{ Polymeric NPs: } \\
\hline Starches & TLRs, CLRs, RLRs & NF-kB & $\begin{array}{l}\text { IL-6, IL-1 } \beta, \text { TNF- } \alpha, \text { CCL2, } \\
\text { CCL5, CCL4 }\end{array}$ & $\begin{array}{l}{[15],[16]} \\
{[17]}\end{array}$ \\
\hline Chitin/Chitosan & TLR2, CD206, Dectin 1 & ERK, NF-KB, MAPK & IL-2, IFN-y, IL-10, IL-6, IL-8 & [19], [24] \\
\hline PLGA & Multiple PRRs & ERK, NF-KB, MHC-1 & $\begin{array}{l}\text { IL-6, IL-1 } \beta, \text { TNF- } \alpha, \text { IL-2, } \\
\text { IFN- } \gamma, \text { IL-10 }\end{array}$ & [27], [28] \\
\hline \multicolumn{5}{|l|}{ Protein NPs: } \\
\hline Virus like particles & TLR7, TLR9, PTX & $\begin{array}{l}\text { TGF- } \beta \text {, MHC-1, Lectin/classical } \\
\text { complement pathways, MAPK, } \\
\text { NF-kB }\end{array}$ & IL-1 $\beta$, TNF- $\alpha$, CCL2, IL-6 & [49] \\
\hline \multicolumn{5}{|l|}{ LNPs: } \\
\hline $\begin{array}{l}\text { Liposomes and } \\
\text { SLNPs }\end{array}$ & TLRs, CD206 & $\begin{array}{l}\text { ERK, MAPK, classical pathway } \\
\text { binding to IgG, IgM, C1q, CRP }\end{array}$ & CCL2, IFN-y, IL-17A, IL-17F & [59], [86] \\
\hline
\end{tabular}

\section{Conclusion}

Several formulations are increasingly taking advantages of nanotechnology. In particular, biodegradable NPs reveal huge versatility of employment in medicine. The possibility to design immune-compatible nano-tools will allow the development of highly performing gene or drug delivery systems. Vaccine adjuvant technology could also be improved by the use of degradable NPs eliciting tailored immune responses. Nevertheless, the fine tuning of the IS must be carefully controlled and continuous immunological research will be needed to face the amazing growing development of NM. In particular, NP surface chemistry and its modifications to improve solubility and delayed clearance must be deeply investigated as suggested by antibody-mediated uncontrolled immune responses to PEGylated NPs already used in nanomedications.

\section{Abbreviations}

CARPA: complement-activation-related pseudo-allergy

CCL: C-C motif ligand

CD: cluster of differentiation

DCs: dendritic cells

ERK: extracellular regulated kinase

IFN: interferons

Ig: immunoglobulin

IL: interleukin

IS: immune system

LNPs: lipid nanoparticles

MAPK: mitogen-activated protein kinase

MHC: major histocompatibility complex

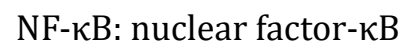

NMs: nanomaterials

NPs: nanoparticles

PC: protein corona

PEG: poly(ethylene glycol)

PLGA: poly (lactic-co-glycolic acid)

PRRs: protein recognition receptors 
SLNPs: solid lipid nanoparticles

Th: T helper (lymphocytes)

TLRs: Toll-like receptors

TNF: tumor necrosis factor

VLPs: virus like particles

\section{Declarations}

Author contributions

AP wrote the manuscript; GB conceived, wrote, and revised the manuscript.

\section{Conflicts of interest}

The authors declare that they have no conflicts of interest.

\section{Ethical approval}

Not applicable.

\section{Consent to participate}

Not applicable.

\section{Consent to publication}

Not applicable.

Availability of data and materials

Not applicable.

\section{Funding}

Not applicable.

\section{Copyright}

(C) The Author(s) 2021.

\section{References}

1. Abdullaeva Z, Abdullaeva Z. Nanomaterials in medicine. In: Nanomaterials in Daily Life. Berlin: Springer International Publishing; 2017.

2. Perioli L, Pagano C, Ceccarini MR. Current highlights about the safety of inorganic nanomaterials in healthcare. Curr Med Chem. 2018;26:2147-65.

3. Gatto F, Bardi G, Gatto F, Bardi G. Metallic nanoparticles: general research approaches to immunological characterization. Nanomaterials. 2018;8:753.

4. Gamucci O, Bertero A, Gagliardi M, Bardi G. Biomedical nanoparticles: overview of their surface immunecompatibility. Coatings. 2014;4:139-59.

5. Fam SY, Chee CF, Yong CY, Ho KL, Mariatulqabtiah AR, Tan WS. Stealth coating of nanoparticles in drugdelivery systems. Nanomaterials. 2020;10:787.

6. Johnson L, Duschl A, Himly M. Nanotechnology-based vaccines for allergen-specific immunotherapy: potentials and challenges of conventional and novel adjuvants under research. Vaccines (Basel). 2020;8:237.

7. Feng X, Xu W, Li Z, Song W, Ding J, Chen X. Immunomodulatory Nanosystems. Adv Sci. 2019;6:1900101.

8. Zhou J, Krishnan N, Jiang Y, Fang RH, Zhang L. Nanotechnology for virus treatment. Nano Today. 2021;36:101031. 
9. Varahachalam SP, Lahooti B, Chamaneh M, Bagchi S, Chhibber T, Morris K, et al. Nanomedicine for the SARS-CoV-2: state-of-the-art and future prospects. Int J Nanomedicine. 2021;16:539-60.

10. Pisani A, Pompa PP, Bardi G. Potential applications of nanomaterials to quench the cytokine storm in coronavirus disease 19. Front Bioeng Biotechnol. 2020;8:906.

11. Olatunji O. Classification of natural polymers. In: Natural Polymers. Berlin: Springer International Publishing; 2015. pp. 1-17.

12. Tian H, Tang Z, Zhuang X, Chen X, Jing X. Biodegradable synthetic polymers: Preparation, functionalization and biomedical application. Prog Polym Sci. 2012;37:237-80.

13. Torres FG, Troncoso OP, Pisani A, Gatto F, Bardi G. Natural polysaccharide nanomaterials: An overview of their immunological properties. Int J Mol Sci. 2019;20:5092.

14. Gatto F, Troncoso OP, Brunetti V, Malvindi MA, Pompa PP, Torres FG, et al. Human monocyte response to Andean-native starch nanoparticles. Starch. 2016;68:1016-23.

15. Torres FG, Troncoso OP, Gamucci O, Corvaglia S, Brunetti V, Bardi G. Immunological properties of Andean starch films are independent of their nanometric roughness and stiffness. Int J Biol Macromol. 2015;75:460-6.

16. Troncoso OP, Torres FG. Non-conventional starch nanoparticles for drug delivery applications. Med DEVICES SENSORS. 2020;3:e10111.

17. Marques AP, Reis RL, Hunt JA. Cytokine secretion from mononuclear cells cultured in vitro with starchbased polymers and poly-L-lactide. J Biomed Mater Res A. 2004;71:419-29.

18. Fuchs K, Cardona Gloria Y, Wolz O, Herster F, Sharma L, Dillen CA, et al. The fungal ligand chitin directly binds TLR 2 and triggers inflammation dependent on oligomer size. EMBO Rep. 2018;19:e46065.

19. Elieh Ali Komi D, Sharma L, Dela Cruz CS. Chitin and its effects on inflammatory and immune responses. Clin Rev Allergy Immunol. 2018;54:213-23.

20. Jhaveri J, Raichura Z, Khan T, Momin M, Omri A. Chitosan Nanoparticles-insight into properties, functionalization and applications in drug delivery and theranostics. Molecules. 2021;26:272.

21. Wen ZS, Xu YL, Zou XT, Xu ZR. Chitosan nanoparticles act as an adjuvant to promote both Th1 and Th2 immune responses induced by ovalbumin in mice. Mar Drugs. 2011;9:1038-55.

22. Sun B, Yu S, Zhao D, Guo S, Wang X, Zhao K. Polysaccharides as vaccine adjuvants. Vaccine. 2018;36: 5226-34.

23. Shariatinia Z. Pharmaceutical applications of chitosan. Adv Colloid Interface Sci. 2019;137131-94.

24. Farhadihosseinabadi B, Zarebkohan A, Eftekhary M, Heiat M, Moosazadeh Moghaddam M, Gholipourmalekabadi M. Crosstalk between chitosan and cell signaling pathways. Cell Mol Life Sci. 2019;76:2697-718.

25. Connor EF, Lees I, Maclean D. Polymers as drugs-Advances in therapeutic applications of polymer binding agents. J Polym Sci Part A Polym Chem. 2017;55:3146-57.

26. Marin E, Briceño MI, Caballero-George C. Critical evaluation of biodegradable polymers used in nanodrugs. Int J Nanomedicine. 2013;8:3071-91.

27. Danhier F, Ansorena E, Silva JM, Coco R, Le Breton A, Préat V. PLGA-based nanoparticles: an overview of biomedical applications. J Control Release. 2012;161:505-22.

28. Mir M, Ahmed N, Rehman AU. Recent applications of PLGA based nanostructures in drug delivery. Colloids Surf B Biointerfaces. 2017;159:217-31.

29. Nicolete R, Santos DFD, Faccioli LH. The uptake of PLGA micro or nanoparticles by macrophages provokes distinct in vitro inflammatory response. Int Immunopharmacol. 2011;11:1557-63.

30. Megy S, Aguero S, Da Costa D, Lamrayah M, Berthet M, Primard C, et al. Molecular dynamics studies of poly(Lactic acid) nanoparticles and their interactions with vitamin E and TLR agonists Pam1CSK4 and Pam3CSK4. Nanomaterials. 2020;10:2209. 
31. Martínez-Negro M, González-Rubio G, Aicart E, Landfester K, Guerrero-Martínez A, Junquera E. Insights into colloidal nanoparticle-protein corona interactions for nanomedicine applications. Adv Colloid Interface Sci. 2021;289:102366.

32. Gessner A, Lieske A, Paulke BR, Müller RH. Functional groups on polystyrene model nanoparticles: Influence on protein adsorption. J Biomed Mater Res A. 2003;65:319-26.

33. Gessner A, Lieske A, Paulke B, Müller R. Influence of surface charge density on protein adsorption on polymeric nanoparticles: analysis by two-dimensional electrophoresis. Eur J Pharm Biopharm. 2002;54:165-70.

34. Cagliani R, Gatto F, Bardi G. Protein Adsorption: a feasible method for nanoparticle functionalization? Materials (Basel). 2019;12:1991.

35. Bros M, Nuhn L, Simon J, Moll L, Mailänder V, Landfester K, et al. The protein corona as a confounding variable of nanoparticle-mediated targeted vaccine delivery. Front Immunol. 2018;9:1760.

36. Neagu M, Piperigkou Z, Karamanou K, Engin AB, Docea AO, Constantin C, et al. Protein bio-corona: critical issue in immune nanotoxicology. Arch Toxicol. 2017;91:1031-48.

37. Moghimi SM, Simberg D. Complement activation turnover on surfaces of nanoparticles. Nano Today. 2017;15:8-10.

38. Chen F, Wang G, Griffin JI, Brenneman B, Banda NK, Holers VM, et al. Complement proteins bind to nanoparticle protein corona and undergo dynamic exchange in vivo. Nat Nanotechnol. 2017;12:387-93.

39. Vu VP, Gifford GB, Chen F, Benasutti H, Wang G, Groman E V., et al. Immunoglobulin deposition on biomolecule corona determines complement opsonization efficiency of preclinical and clinical nanoparticles. Nat Nanotechnol. 2019;14:260-8.

40. Suk JS, Xu Q, Kim N, Hanes J, Ensign LM. PEGylation as a strategy for improving nanoparticle-based drug and gene delivery. Adv Drug Deliv Rev. 2016;99:28-51.

41. Kozma GT, Shimizu T, Ishida T, Szebeni J. Anti-PEG antibodies: properties, formation, testing and role in adverse immune reactions to PEGylated nano-biopharmaceuticals. Adv Drug Deliv Rev. 2020;154155:163-75.

42. Hong L, Wang Z, Wei X, Shi J, Li C. Antibodies against polyethylene glycol in human blood: a literature review. J Pharmacol Toxicol Methods. 2020;102:106678.

43. Zhang P, Sun F, Liu S, Jiang S. Anti-PEG antibodies in the clinic: current issues and beyond PEGylation. J Control Release. 2016;244:184-93.

44. Yang Q, Jacobs TM, McCallen JD, Moore DT, Huckaby JT, Edelstein JN, et al. Analysis of pre-existing IgG and IgM antibodies against polyethylene glycol (PEG) in the general population. Anal Chem. 2016;88: 11804-12.

45. Stone CA, Liu Y, Relling MV, Krantz MS, Pratt AL, Abreo A, et al. Immediate hypersensitivity to polyethylene glycols and polysorbates: more common than we have recognized. J Allergy Clin Immunol Pract. 2019;7:1533-40.

46. Neun BW, Barenholz Y, Szebeni J, Dobrovolskaia MA. Understanding the role of anti-PEG antibodies in the complement activation by Doxil in vitro. Molecules. 2018;23:1700.

47. Thi TTH, Pilkington EH, Nguyen DH, Lee JS, Park KD, Truong NP. The importance of poly(ethylene glycol) alternatives for overcoming PEG immunogenicity in drug delivery and bioconjugation. Polymers. 2020;12:298.

48. Jain A, Singh SK, Arya SK, Kundu SC, Kapoor S. Protein nanoparticles: promising platforms for drug delivery applications. ACS Biomater Sci Eng. 2018;4:3939-61.

49. Mohsen MO, Gomes AC, Vogel M, Bachmann MF. Interaction of viral capsid-derived virus-like particles (VLPs) with the innate immune system. Vaccines. 2018;6:37. 
50. Bardi G. Nanometric virus-like particles: key tools for vaccine and adjuvant technology. Vaccines. 2020;8:430.

51. Dedeo CL, Teschke CM, Alexandrescu AT. Keeping it together: structures, functions, and applications of viral decoration proteins. Viruses. 2020;12:1163.

52. Papini E, Tavano R, Mancin F. Opsonins and dysopsonins of nanoparticles: facts, concepts, and methodological guidelines. Front Immunol. 2020;11:567365.

53. Pasto A, Giordano F, Evangelopoulos M, Amadori A, Tasciotti E. Cell membrane protein functionalization of nanoparticles as a new tumor-targeting strategy. Clin Transl Med. 2019;8:8.

54. Cagliani R, Gatto F, Cibecchini G, Marotta R, Catalano F, Sanchez-Moreno P, et al. CXCL5 modified nanoparticle surface improves CXCR2 ${ }^{+}$cell selective internalization. Cells. 2019;9:56.

55. Pisani A, Donno R, Gennari A, Cibecchini G, Catalano F, Marotta R, etal.CXCL12-PLGA/pluronicnanoparticle internalization abrogates CXCR4-mediated cell migration. Nanomater (Basel). 2020;10:2304.

56. Legler DF, Thelen M. Chemokines: chemistry, biochemistry and biological function. Chimia (Aarau). 2016;70:856-9.

57. Bangham AD, Standish MM, Watkins JC. Diffusion of univalent ions across the lamellae of swollen phospholipids. J Mol Biol. 1965;13:238-52.

58. Sharma A, Sharma US. Liposomes in drug delivery: progress and limitations. Int J Pharm. 1997;154: 123-40.

59. Zahednezhad F, Saadat M, Valizadeh H, Zakeri-Milani P, Baradaran B. Liposome and immune system interplay: challenges and potentials. J Control Release. 2019;305:194-209.

60. Allen TM, Chonn A. Large unilamellar liposomes with low uptake into the reticuloendothelial system. FEBS Lett. 1987;223:42-6.

61. Allen TM, Hansen C, Rutledge J. Liposomes with prolonged circulation times: factors affecting uptake by reticuloendothelial and other tissues. Biochim Biophys Acta. 1989;981:27-35.

62. Allen TM, Austin GA, Chonn A, Lin L, Lee KC. Uptake of liposomes by cultured mouse bone marrow macrophages: influence of liposome composition and size. Biochim Biophys Acta. 1991;1061:56-64.

63. Chonn A, Cullis PR, Devine DV. The role of surface charge in the activation of the classical and alternative pathways of complement by liposomes. J Immunol. 1991;146:4234-41.

64. Aramaki Y, Akiyama K, Hara T, Tsuchiya S. Recognition of charged liposomes by rat peritoneal and splenic macrophages: effects of fibronectin on the uptake of charged liposomes. Eur J Pharm Sci. 1995;3:63-70.

65. Moein Moghimi S, Patel HM. Serum opsonins and phagocytosis of saturated and unsaturated phospholipid liposomes. Biochim Biophys Acta. 1989;984:384-7.

66. Moein Moghimi S, Patel HM. Opsonophagocytosis of liposomes by peritoneal macrophages and bone marrow reticuloendothelial cells. Biochim Biophys Acta. 1992;1135:269-74.

67. Funato K, Yoda R, Kiwada H. Contribution of complement system on destabilization of liposomes composed of hydrogenated egg phosphatidylcholine in rat fresh plasma. Biochim Biophys Acta. 1992;1103:198-204.

68. Ishida T, Yasukawa K, Kojima H, Harashima H, Kiwada $\mathrm{H}$. Effect of cholesterol content in activation of the classical versus the alternative pathway of rat complement system induced by hydrogenated egg phosphatidylcholine-based liposomes. Int J Pharm. 2001;224:69-79.

69. Huong TM, Harashima H, Kiwada H. Complement dependent and independent liposome uptake by peritoneal macrophages: cholesterol content dependency. Biol Pharm Bull. 1998;21:969-73.

70. Huong TM, Ishida T, Harashima H, Kiwada H. Species difference in correlation between in vivo/in vitro liposome-complement interactions. Biol Pharm Bull 2001;24:439-41.

71. Mohamed M, Abu Lila AS, Shimizu T, Alaaeldin E, Hussein A, Sarhan HA, et al. PEGylated liposomes: immunological responses. Sci Technol Adv Mater. 201920:710-24. 
72. Varypataki EM, van der Maaden K, Bouwstra J, Ossendorp F, Jiskoot W. Cationic liposomes loaded with a synthetic long peptide and poly(I:C): a defined adjuvanted vaccine for induction of antigen-specific $\mathrm{T}$ cell cytotoxicity. AAPS J. 2015;17:216-26.

73. Varypataki EM, Benne N, Bouwstra J, Jiskoot W, Ossendorp F. Efficient eradication of established tumors in mice with cationic liposome-based synthetic long-peptide vaccines. Cancer Immunol Res. 2017;5:222-33.

74. Hattori Y, Yamashita J, Sakaida C, Kawano K, Yonemochi E. Evaluation of antitumor effect of zoledronic acid entrapped in folate-linked liposome for targeting to tumor-associated macrophages. J Liposome Res. 2015;25:131-40.

75. Burke ML, Veer M De, Pleasance J, Neeland M, Elhay M, Harrison P, et al. Innate immune pathways in afferent lymph following vaccination with poly(I:C)-containing liposomes. Innate Immun. 2014;20: 501-10.

76. Abhyankar MM, Noor Z, Tomai MA, Elvecrog J, Fox CB, Petri WA. Nanoformulation of synergistic TLR ligands to enhance vaccination against Entamoeba histolytica. Vaccine. 2017;35:916-22.

77. Li P, Chen S, Jiang Y, Jiang J, Zhang Z, Sun X. Dendritic cell targeted liposomes-protamine-DNA complexes mediated by synthetic mannosylated cholestrol as a potential carrier for DNA vaccine. Nanotechnology. 2013;24:295101.

78. Sahin U, Muik A, Derhovanessian E, Vogler I, Kranz LM, Vormehr M, et al. Publisher correction: COVID-19 vaccine BNT162b1 elicits human antibody and TH1 T cell responses. Nature. 2021;590:E17.

79. Sahin U, Muik A, Derhovanessian E, Vogler I, Kranz LM, Vormehr M, et al. COVID-19 vaccine BNT162b1 elicits human antibody and TH1 T cell responses. Nature. 2020;586:594-9.

80. Mulligan MJ, Lyke KE, Kitchin N, Absalon J, Gurtman A, Lockhart S, et al. Phase I/II study of COVID-19 RNA vaccine BNT162b1 in adults. Nature. 2020;586:589-93.

81. Hiller N, Goldberg SN, Cohen-Cymberknoh M, Vainstein V, Simanovsky N. Lymphadenopathy associated with the COVID-19 vaccine. Cureus. 2021;13:e13524.

82. Klimek L, Novak N, Cabanillas B, Jutel M, Bousquet J, Akdis CA. Allergenic components of the mRNA1273 vaccine for COVID-19: possible involvement of polyethylene glycol and IgG-mediated complement activation. Allergy. Forthcoming 2021.

83. Lokerse WJM, Lazarian A, Kleinhempel A, Petrini M, Schwarz P, Hossann M, et al. Mechanistic investigation of thermosensitive liposome immunogenicity and understanding the drivers for circulation half-life: a polyethylene glycol versus 1,2-dipalmitoyl-sn-glycero-3-phosphodiglycerol study. J Control Release. 2021;333:1-15.

84. Samaridou E, Heyes J, Lutwyche P. Lipid nanoparticles for nucleic acid delivery: Current perspectives. Adv Drug Deliv Rev. 2020;154-155:37-63.

85. Moss KH, Popova P, Hadrup SR, Astakhova K, Taskova M. Lipid nanoparticles for delivery of therapeutic RNA oligonucleotides. Mol Pharm. 2019;16:2265-77.

86. Mehnert W, Mäder K. Solid lipid nanoparticles: production, characterization and applications. Adv Drug Deliv Rev. 2001;47:165-96.

87. Lutz J, Lazzaro S, Habbeddine M, Schmidt KE, Baumhof P, Mui BL, et al. Unmodified mRNA in LNPs constitutes a competitive technology for prophylactic vaccines. NPJ Vaccines. 2017;2:29.

88. Tabernero J, Shapiro GI, LoRusso PM, Cervantes A, Schwartz GK, Weiss GJ, et al. First-in-humans trial of an RNA interference therapeutic targeting VEGF and KSP in cancer patients with liver involvement. Cancer Discov. 2013;3:406-17.

89. Semple SC, Klimuk SK, Harasym TO, Dos Santos N, Ansell SM, Wong KF, et al. Efficient encapsulation of antisense oligonucleotides in lipid vesicles using ionizable aminolipids: formation of novel small multilamellar vesicle structures. Biochim Biophys Acta. 2001;1510:152-66. 
90. Sedic M, Senn JJ, Lynn A, Laska M, Smith M, Platz SJ, et al. Safety evaluation of lipid nanoparticleformulated modified mRNA in the sprague-dawley rat and cynomolgus monkey. Vet Pathol. 2018;55: 341-54.

91. Kumar V, Qin J, Jiang Y, Duncan RG, Brigham B, Fishman S, et al. Shielding of lipid nanoparticles for siRNA delivery: impact on physicochemical properties, cytokine induction, and efficacy. Mol Ther Nucleic Acids. 2014;3:e210. 